\title{
A probabilistic approach to sea level rise up to the year 2100 at Kołobrzeg, Poland
}

\author{
Andrzej Wróblewski* \\ Institute of Oceanology PAS, 81-712 Sopot, Poland
}

\begin{abstract}
As an example of the linear forecast method, the application of a linear trend to annual mean sea level data from Kołobrzeg is analysed, and the mean sea level rise for a 110 yr time period is forecast. On the basis of the classical trend theory, the possibility of making a linear forecast on the basis of variable confidence intervals is demonstrated and various computational techniques are compared. The method assumes the application of confidence intervals not only to the stochastic component of the forecast model but also to the estimated trend parameters. As a result, depending on the assumptions and measurement data, the linear forecast method can be adapted to a variety of situations where low-lying areas are under threat from rising sea levels. The possibility of including the mean sea level rise forecast in the probabilistic forecast of storm surges is discussed. Example calculations for the period 1901 to 1990 were performed for a time series of maximum annual sea levels at Kołobrzeg, estimated by Gumbel's distribution.
\end{abstract}

KEY WORDS: Sea level rise - Sea level forecast · Storm surges · Greenhouse effect

Resale or republication not permitted without written consent of the publisher

\section{INTRODUCTION}

The global rise in mean sea level, a problem generally associated with the climatic changes brought about by the greenhouse effect, is now being seen as an extremely serious threat to low-lying coastal areas. The rise in the level of the world ocean has been proven, although estimates of the scale of the phenomenon vary widely. This difficulty hinges on the fact that tide gauge readings show only relative changes, which depend not only on actual variations in sea level but also on the vertical movements of the Earth's crust in the region of the relevant tide gauge. The necessary corrections to sea level readings were made following the publication of the theory of vertical post-glacial tectonic movements (Tushingham \& Peltier 1991). However, it is hard to make estimates of the vertical movements of the Earth's crust with an accuracy comparable to that of oceanographic measurements. This applies in particular to historical time series. If we take into account just the readings of tide gauges where measurable changes in the zero position have oc-

*E-mail: wroblew@iopan.gda.pl curred, we have a global sea level rise of $18 \mathrm{~cm}$ over the last 100 yr (Douglas 1991). According to the more extensive IPCC (Intergovernmental Panel on Climate Changes) studies, however, the range of uncertainty of this estimate is 10 to $25 \mathrm{~cm}$ (Warrick et al. 1996). This same source puts the 'best estimate' of mean global sea level rise for the year 2100 at $49 \mathrm{~cm}$ with a 20 to $86 \mathrm{~cm}$ range of uncertainty. The crucial factors in any estimate of sea level rise may be those resulting from global warming, i.e. the thermal expansion of water, as well as the melting of glaciers, ice caps, and the circumpolar ice sheets. It is generally thought that the global rise is not just a simple reflection of the variations in sea level at particular tide gauges. Indeed, in some places, a drop in sea level can be expected as a consequence of changes in atmospheric circulation and water dynamics.

When we are considering a local, statistical forecast of mean sea level, the main problem is establishing the trend of relative sea level rise, in other words, establishing whether this rise is linear. The division of sea level changes into those due to vertical shifts in the position of the tide gauge zero and those caused by real sea level variations is of minor importance in many 
cases. Tectonic movements merely contribute one further aspect to the uncertainty of the future relative sea level. Computations have demonstrated that neither in the world ocean nor in European seas (Woodworth 1990, Gornitz \& Solov 1991, Douglas 1992) has there been any acceleration in sea level rise in the 20th century. It is generally accepted that a linear trend best approximates the rise, and that deviations from this linearity will be strictly localised.

Based on global warming and the resulting factors, IPCC forecasts for the 21st century predict a rise in sea level ca 3 times greater than that which occurred in the 20th century. Thus, an extrapolation of the linear trend recorded so far will underestimate forecasts. Nevertheless, statistical forecasts must be drawn up, as they are of the utmost importance in the design of hydrotechnical structures. In most countries, such structures are built on the basis of normative return periods of storm sea levels and their error bounds. The lifetime of hydrotechnical structures, their capital depreciation period and the disastrous consequences of their being inundated by the sea impose the need to produce forecasts for longer periods of time. The present paper discusses the possible application of the linear forecast method to the mean sea level rise in a tideless sea on the basis of computations carried out for the Kołobrzeg, Poland (southern Baltic Sea) tide gauge data (Dziadziuszko 1994). The principal problem with a linear trend forecast is that only if the confidence intervals of the trend are enlarged will it be possible to compute predicted values within the IPCC limits. Analysis of the confidence intervals also enables the effect of the predicted mean sea levels to be introduced into the probabilistic computation of maximum storm sea levels. The present paper provides a new, predictive approach which can be treated as an extension of the author's previous paper (Wróblewski 1994).

\section{COMPUTATION OF THE FORECAST BY EXTRAPOLATING THE LINEAR TREND}

Long-term sea level variability was analysed on the basis of mean annual values. Computing annual sea level data by employing the monthly mean of readings is equivalent to applying lowpass filtration. For monthly mean filtering the maximum aliasing error is assessed at $0.055 \%$ of the $\mathrm{M}_{2}$ amplitude (Pugh 1987). Along the Polish coast the Baltic Sea is practically tideless; thus oscillation periods of lesser significance, shorter than annual period, have been ignored. The aliasing error of the annual mean is without practical meaning. If reliable data on the vertical movements of the tide gauge zero are available, the forecast should be computed separately for $\xi_{\mathrm{r}}(t)$ and $\xi_{\mathrm{M}}(t)$ by the equation:

$$
\xi(t)=\xi_{\mathrm{r}}(t) \pm \xi_{\mathrm{M}}(t)
$$

where $\xi(t)=$ the relative annual mean sea level recorded at the tide gauge; $\xi_{\mathrm{r}}(t)=$ the real annual mean sea level; $\xi_{\mathrm{M}}(t)=$ the apparent annual mean sea level caused by tectonic movements $t=1,2 \ldots \ldots \ldots \ldots, N$, the measurement time variable

In Eq. (1) the final result will be the superposition of both components. But in the particular case of Kołobrzeg it was not possible to separate the components of the relative sea level, so $\xi(t)$ was calculated on the basis of the linear trend forecast and the estimates of trend parameters using the least squares method (Draper \& Smith 1981, Zelias 1984). Sea levels are presented in Fig. 1 and given by the equation:

$$
\xi(t)=\mathbf{c}^{\prime} \hat{\mathbf{a}} \pm u(t)
$$

where $\mathbf{c}^{\prime}=$ the transpose of the time variable vector, $\mathbf{c}=\left[\begin{array}{l}1 \\ \mathrm{~N}\end{array}\right]_{;} \hat{\mathbf{a}}=$ the estimated value of the trend parameter vector, $\mathbf{a}=\left[\begin{array}{l}\mathrm{a}_{0} \\ \mathrm{a}_{1}\end{array}\right] ;$ and $u(t)=$ the random component.

In order to ascertain whether the assumed linear form of the trend does in fact correspond with the data under consideration, a new series of increments was computed with the aid of the equation:

$$
\xi^{*}(t)=\Delta \xi(t)
$$

where $\xi^{*}(t)=$ the increment series, and $\Delta=$ the backward difference operator.

If the trend is linear, the series should be random, i.e. parameter $\hat{a}_{1}$ of $\xi^{*}(t)$ should be zero in value. Application of Student's test at the $5 \%$ significance level indicated that parameter $\hat{a}_{1}$, estimated for the series $\xi^{*}(t)$, does not differ significantly from zero; thus, the trend equation has been chosen correctly. Furthermore,

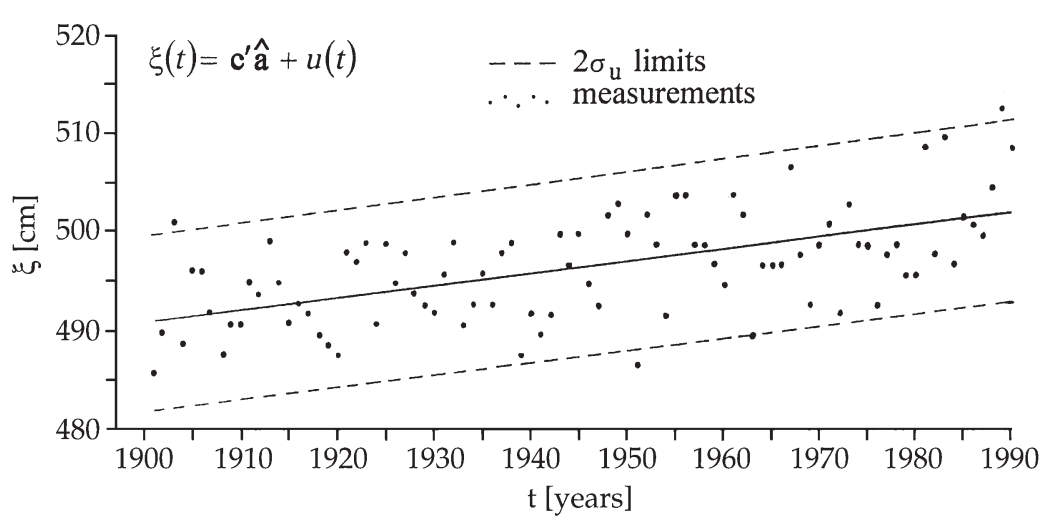

Fig. 1. Linear trend in mean annual sea levels at Kołobrzeg. Tide gauge readings for 1901 to 1990 
Anderson's test for a probability of 0.95 showed the random component $u(t)$ given in Eq. (2) to be independent. Finally, at the 0.98 probability level, the distribution of the $u(t)$ series was found to be normal (Snedecor \& Cochran 1967). It should be emphasised that the results of testing presented here are necessary conditions for the application of the proposed estimation technique. If the variety of factors forcing sea level changes is taken into consideration, the proven normality of the series is in accordance with the central limit theorem.

When the trend equation is extrapolated to obtain the forecast maximum lead time $T_{\mathrm{m}}$, the forecast is given by the equation:

$$
\hat{\xi}_{T_{\mathrm{m}}}=\mathbf{C}^{\prime} \hat{\mathbf{a}}
$$

where the forecast maximum lead time $T_{\mathrm{m}}=N+1$, $N+2, N+\ldots ; \mathbf{c}^{\prime}=$ the transpose of the time variable vector, $\mathbf{C}=\left[\frac{1}{T_{\mathrm{m}}}\right]$.

Then the forecast error in Eq. (4) is presented by the equation:

$$
D_{T_{\mathrm{m}}}=\hat{\xi}_{T_{\mathrm{m}}}-\xi_{T_{\mathrm{m}}}
$$

where $D_{T_{\mathrm{m}}}=$ the forecast error at time $T_{\mathrm{m}}$, and $\xi_{T_{\mathrm{m}}}=$ the real sea level at time $T_{\mathrm{m}}$.

Given the theoretical assumptions $E(\hat{\mathbf{a}})=\mathbf{a}$ and $E\left(u_{T_{\mathrm{m}}}\right)=0$, the forecast error is presented by:

$$
E\left(D_{T_{\mathrm{m}}}\right)=E\left(\hat{\xi}_{T_{\mathrm{m}}}-\xi_{T_{\mathrm{m}}}\right)=E\left[\mathbf{c}^{\prime}(\hat{\mathbf{a}}-\mathbf{a})-u_{T_{\mathrm{m}}}\right]=0
$$

where $u_{T_{\mathrm{m}}}=$ the random component at time $T_{\mathrm{m}}$.

When computing forecasts from Eq. (4), estimated values of $\hat{\mathbf{a}}$ are used in place of their real values a. By doing this, the source of forecast errors in Eq. (5) includes the errors in estimating the parameters of a sample and the unknown value of $u_{T_{\mathrm{m}}}$. It should be stressed that for very large values of $N$, theoretically for $N \rightarrow \infty$, the variance of the random component is the lower limit of the variance of the forecast error.

In deriving the variance of $D_{T_{\mathrm{m}}}$ (taking into account the variance of the parameters, their covariance and the variance of the random component) one arrives at the equation:

where $\quad \Phi^{\prime} \Phi=\left[\begin{array}{c}N \sum_{1}^{N} t \\ \sum_{1}^{N} t \sum_{1}^{N} t^{2}\end{array}\right]$

$D_{T_{\mathrm{m}}}^{2}=$ the variance of the forecast error at time $T_{\mathrm{m}}$; $\sigma_{u}^{2}=$ the variance of the random component $u(t)$.

To simplify the notation, the matrix component of Eq. (7) will henceforth be denoted by $\gamma$ in accordance with:

$$
\gamma=1+\mathbf{c}^{\prime}\left(\Phi^{\prime} \Phi\right)^{-1} \mathbf{C}
$$

$D_{T_{\mathrm{m}}}$ denotes the mean forecast error calculated as the standard deviation of the variable $u(t)$ (in the absence of other data) multiplied by $\gamma^{1 / 2}$. The mathematical transformations are given in the literature previously cited for Eq. (2). The best way of obtaining greater forecast errors would be to introduce confidence intervals for $D_{T_{\mathrm{m}}}^{2}$ in Eq. (7) and consequently in Eq. (10), but the interval limits are too narrow for this purpose. In order to forecast high sea level rises we have of necessity to assume that $\sigma_{u}$ is taken to be the upper limit of the normal confidence interval; hence, $u_{T_{\mathrm{m}}}$ lies within the interval $\left\langle\bar{u}-\sigma_{u}, \bar{u}+\sigma_{u}\right\rangle$ with a probability of 0.68 . The normality of the series under consideration has already been proven. Higher values of the upper limit of occurrence of the variable are presented in the same way. Forecasting extremely high sea level rises is feasible if we assume a probability for $u_{T_{\mathrm{m}}}>3 \sigma_{u}$ according to Eq. (7); this however, is rarely used owing to the Chebyshev inequality.

As an alternative to Eq. (7), one can calculate the mean forecast error on the assumption that the only source of error is the random component $u(t)$. This assumption is all the more probable the longer the measurement series that has been used in the estimation of parameters. As has already been mentioned, such an approach is associated with the lower limit of variance given by Eq. (7). In this case, the component $\gamma$ in the equation is ignored. $\gamma$ depends only on the forecast extrapolation time and on the series of the variable $t$. Thus, it is independent of the recorded values of $\xi(t)$. For rational extrapolation times, not greater than $120 \%$ of the measured data number, this component does not much increase values computed using Eq. (7) (Fig. 2).

These are not the only possibilities for adapting the linear trend forecast to the expected considerable sea

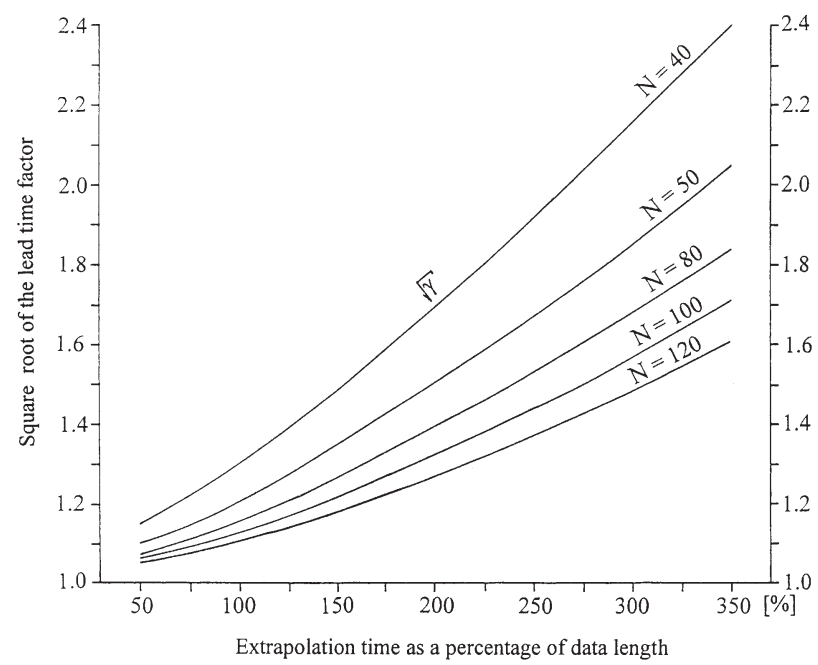

Fig. 2. Values of $\gamma$ with respect to length of data series and forecast lead time 
level rises. Estimation of the trend equation parameters could be linked with the confidence level $\psi$. If we assume the upper limits of these confidence intervals according to the Student's distribution, the forecast values can be very much larger for high interval probabilities. Given the above assumptions the final forecast is presented by the equation:

$$
\hat{\xi}_{T_{\mathrm{m} \alpha \psi}}=\mathbf{c}^{\prime} \hat{\mathbf{a}}_{\psi}+\hat{D}_{T_{\mathrm{m} \alpha}}
$$

where $\hat{D}_{T_{\mathrm{m} \alpha}}=$ the forecast error with probability $1-\alpha$ since $u_{T_{\mathrm{m} \alpha}}$ is the upper limit of the normal interval of $u(t)$ for confidence $\alpha_{;} \mathbf{a}_{\psi}=$ vector $\mathbf{a}$ as the upper limit of the Student's distribution intervals for confidence $\psi$.

These possible applications of the linear trend forecast demonstrate that the forecast depends on subjectively chosen confidence levels. Similar subjective assumptions are used in the case of maximum storm surges, where the sea level return period and the confidence interval of the extreme quantiles are always imposed as standards. The idea of these practical assumptions is to enable the linear forecast model to be applied to the very considerable increase in the mean sea level predicted.

If we wish to compute the forecast for different times on identical principles, once we know the value of $\alpha$ and the longest forecast lead time, it is essential to define the forecast sea level rises when $N<T<T_{\mathrm{m}}$ according to the equations:

$$
\begin{gathered}
\hat{\xi}_{T_{\alpha_{0}}}=\mathbf{c}^{\prime} \hat{\mathbf{a}}+\hat{u}_{T_{\alpha_{0}}} \gamma^{0.5} \\
u_{T_{\alpha_{0}}}=\frac{\hat{\sigma}_{u}+\left(\hat{u}_{T_{\mathrm{m} \alpha}}-\hat{\sigma}_{u}\right)}{\left(T_{\mathrm{m}}-N\right)}(T-N)
\end{gathered}
$$

where $\alpha_{0}=$ the confidence level of $u(t)$ at time $T<T_{\mathrm{m}}$.

One can assume the random part of the forecast error increment to be linear and proportional to the increase in the predicted lead time (see Eq. 12). By
Table 1. Principal characteristics of the linear trend in mean annual sea levels at Kołobrzeg. Tide gauge readings for 1901 to 1990

\begin{tabular}{|lcccc|}
\hline $\begin{array}{l}\hat{\sigma}_{u} \\
(\mathrm{~cm})\end{array}$ & $\begin{array}{c}\hat{a}_{0} \\
(\mathrm{~cm})\end{array}$ & $\begin{array}{c}\hat{a}_{1} \\
\left(\mathrm{~cm} \mathrm{yr}^{-1}\right)\end{array}$ & $\begin{array}{c}\hat{\xi}_{\mathrm{N}} \\
(\mathrm{cm})\end{array}$ & $\begin{array}{c}\hat{\bar{\xi}} \\
(\mathrm{cm})\end{array}$ \\
\hline 4.6 & $491.0 \pm 0.98$ & $0.12 \pm 0.02$ & 501.7 & 496.4 \\
\hline
\end{tabular}

means of this equation we can calculate the random part of the forecast error for an arbitrary lead time $T<$ $T_{\mathrm{m}}$ and read off the confidence level $\alpha^{\mathrm{o}}$ from normal distribution tables. These equations approximate the nearly linear sea level rise curves published by IPCC (Warrick et al. 1996) and increase the forecast error with the increase in risk due to the extension of the forecast lead time. The equations are applicable to engineering structures with a depreciation time shorter than $T_{\mathrm{m}}$.

The principal computation parameters for Kołobrzeg are given in Table 1. Table 2 sets out the computations in which the confidence intervals for the parameters of the distribution and the random component have been used. In accordance with the trend line $(0.12 \pm$ $0.02 \mathrm{~cm} \mathrm{yr}^{-1}$ ), the mean sea level at Kołobrzeg in 1990 was calculated at $502 \mathrm{~cm}$. Assuming the probability of the confidence interval of the parameters to be $1-\psi=0.999$ and that of the random component to be $1-\alpha=0.997$, the mean sea level in 2100 was computed at $547 \mathrm{~cm}$. The rise at Kołobrzeg was $45 \mathrm{~cm}$ $\left(0.41 \mathrm{~cm} \mathrm{yr}^{-1}\right)$, thus very close to the 'best estimate' of $49 \mathrm{~cm}$ published by IPCC. The trend parameters had a significance level $<0.05$. Higher but not calculated forecast rises could be obtained for values of $u_{T_{\mathrm{m} \alpha}}>3 \sigma_{u}$. To facilitate practical applications, one of the aims of this paper was to present the IPCC forecast of the sea level as the probabilistic forecast of the

\begin{tabular}{|c|c|c|c|c|}
\hline $\begin{array}{c}\text { Confidence interval } \\
\text { probability } \\
1-\alpha \text { for } D_{T_{\mathrm{m} \alpha}}\end{array}$ & $\begin{array}{c}\text { Confidence interval } \\
\text { probability } \\
1-\psi \text { for } \hat{\mathbf{a}}\end{array}$ & $\begin{array}{l}\text { Upper limit of } \\
\text { confidence interval } \\
\text { for } D_{T_{\mathrm{m} \alpha}}(\mathrm{cm})\end{array}$ & $\begin{array}{c}\text { Sea level } \hat{\xi}_{T_{\mathrm{m} \psi}}=\mathbf{c}^{\prime} \hat{\mathbf{a}}_{\psi} \\
\text { for upper limit of the } \hat{\mathbf{a}}_{\psi} \\
\text { confidence interval }(\mathbf{c m})\end{array}$ & $\hat{\xi}_{T_{\mathrm{m} \psi}}=\underset{(\mathrm{cm})}{\mathbf{c}^{\prime} \hat{\mathbf{a}}_{\psi}+D_{T_{\mathrm{m} \alpha}}}$ \\
\hline & $\begin{array}{l}- \\
0.95 \\
0.99 \\
0.999\end{array}$ & & $\begin{array}{l}514.9 \\
524.2 \\
527.2 \\
530.9\end{array}$ & \\
\hline $\begin{array}{c}\left(\bar{u}-\sigma_{u,} \bar{u}+\sigma_{u}\right) \\
0.68\end{array}$ & 0.999 & 5.5 & 530.9 & 536 \\
\hline $\begin{array}{c}\left(\bar{u}-2 \sigma_{u,} \bar{u}+2 \sigma_{u}\right) \\
0.95\end{array}$ & 0.999 & 10.9 & 530.9 & 542 \\
\hline $\begin{array}{c}\left(\bar{u}-3 \sigma_{u}, \bar{u}+3 \sigma_{u}\right) \\
0.997\end{array}$ & 0.999 & 16.3 & 530.9 & 547 \\
\hline
\end{tabular}

Table 2. Mean sea level at Kołobrzeg in the year 2100 according to the linear trend forecast for different confidence intervals. Tide gauge readings for 1901 to 1990 
recorded trend. Once the probabilities of the confidence intervals for Kołobrzeg have been established, they can be used to predict the sea level rise at the other tide-gauges along the Polish coast, which display different trends. In this case, engineering designs would need to take into account only the local trend and the same confidence probabilities for the whole coast.

\section{PROBABILISTIC COMPUTATIONS OF HIGH SEA LEVELS}

Storm surge sea level computations were performed for the maximum annual sea levels measured at Kołobrzeg from 1901 to 1990 (Wróblewski 1994) and presented in Fig. 3. Gumbel's distribution (1958) was used to extrapolate these maxima. The uncertainty associated with the factors generating future storm surges is greater than that for the mean sea level rise. This is because of the great difficulties involved in forecasting local gale-force wind conditions, the principal factor forcing sea level rises. The mean sea level forecast was included in the probabilistic computations of maximum levels by superimposing the 2 levels and analysing their confidence limits. This is not the only solution to the problem; other methods of computation have been published, e.g. Bardsley et al. (1990).

Following the introduction of the reduced variable $y$, Gumbel's distribution density function and distribution function are expressed by the equations:

$$
f(y)=\mathrm{e}^{-y-\mathrm{e}^{-y}}
$$

where $y=\beta(\xi-\mu) ; \beta$ and $\mu$ are the parameters of the distribution:

$$
F(y)=\mathrm{e}^{-\mathrm{e}^{-y}}
$$

Adapted for hydrological applications, this is a distribution II of Fisher \& Tippet (1928), which is included

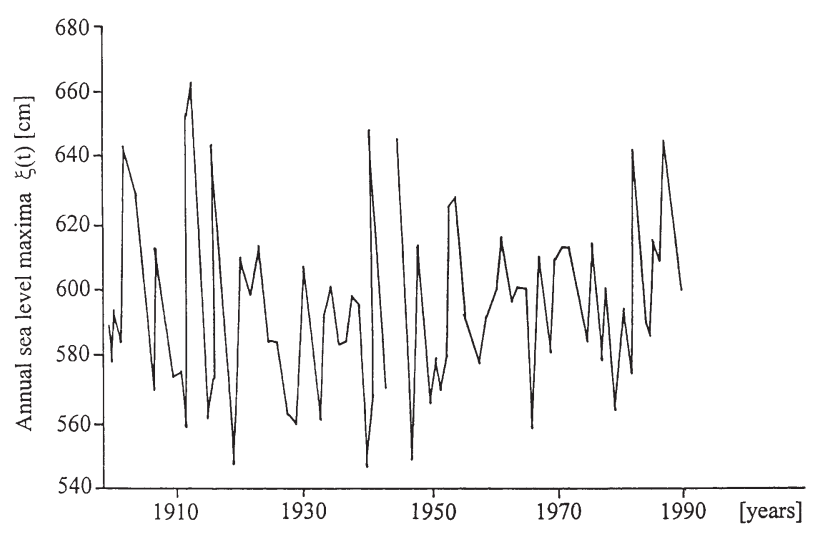

Fig. 3. Annual sea level maxima at Kołobrzeg. Tide gauge readings for 1901 to 1990 in a generalised distribution of extremes (Jenkinson 1955).The parameters of the distribution were calculated using Kimball's (1949) maximum likelihood method given by the equations:

$$
\begin{gathered}
\overline{\mathrm{e}^{-\beta \xi}}-\overline{\beta \xi} \overline{\mathrm{e}^{-\beta \xi}}+\beta \overline{\xi \mathrm{e}^{-\beta \xi}}=0 \\
\mu=-\frac{2.3026 \log (\overline{\mathrm{e}-\beta \xi})}{\beta}
\end{gathered}
$$

Eqs (15) \& (16) were solved iteratively. The results are set out in Table 3, and the confidence intervals resulting from the errors in estimating the parameters in Table 4. In probabilistic computations, the sea level storm surges has been accounted for in that the mean sea level forecast, i.e. c'â, is superimposed on the quantile for the probability of the maximum level distribution (trend subtracted) according to the equation:

$$
\hat{\xi}_{T_{\mathrm{p} \varphi}}=\hat{\xi}_{\mathrm{p} \varphi}+\mathbf{c}^{\prime} \hat{\mathbf{a}}_{T_{\psi}} \pm \hat{D} \xi_{T_{\mathrm{p} \varphi \alpha}}
$$

where $T=$ the forecast time; $\hat{\xi}_{T_{p \varphi}}=$ the estimated sea level maximum quantile at time $T$, the exceeding probability of the quantile is $p$, the probability of the confidence interval for the quantile is $1-\varphi_{i} \hat{\xi}_{p \varphi}=$ estimated quantile (from detrended series); $\psi=$ probability the confidence interval is $1-\psi$ for the vector $\hat{\mathbf{a}} ; \alpha=$ probability the confidence interval is $1-\alpha$ for $u_{T_{\alpha} i}$ $\hat{D} \xi_{T_{p \varphi \alpha}}=$ upper limit of the confidence interval for $\hat{\xi}_{T_{p \varphi}}$ including sea level rise.

The distribution of the mean sea level forecast error $\hat{D}_{T_{\alpha}}$ can be assumed as normal. The error in estimating the quantile of the maximum sea level also has an asymptotically normal distribution for estimates obtained by the maximum likelihood method. For a sample of the size analysed here, this distribution can be regarded as normal. The errors in estimating the mean

Table 3. Probability of sea level maxima at Kołobrzeg computed by Gumbel's method (trend eliminated). Measurements 1901 to 1990 . T: return period; p: exceeding probability

\begin{tabular}{|ccccccccc|}
\hline $\mathrm{p}(\%)$ & 99 & 90 & 80 & 70 & 60 & 50 & 40 & 30 \\
$T(\mathrm{yr})$ & 1.01 & 1.11 & 1.25 & 1.43 & 1.61 & 2.0 & 2.50 & 3.33 \\
$\hat{\xi}(\mathrm{cm})$ & 540 & 556 & 564 & 571 & 577 & 583 & 590 & 598 \\
$\mathrm{p}(\%)$ & 20 & 10 & 5 & 2 & 1 & 0.5 & 0.2 & 0.1 \\
$T(\mathrm{yr})$ & 5 & 10 & 20 & 50 & 100 & 200 & 500 & 1000 \\
$\hat{\xi}(\mathrm{cm})$ & 609 & 626 & 642 & 664 & 680 & 696 & 717 & 733 \\
\hline
\end{tabular}

Table 4. Confidence interval limits for sea level maxima at Kołobrzeg with probability $1-\varphi=0.68$. $\hat{D} \xi_{\mathrm{p} \varphi}$ : upper limit of the $1-\varphi$ confidence interval

\begin{tabular}{|lccccccc|}
\hline $\mathrm{p}(\%)$ & 10 & 5 & 2 & 1 & 0.5 & 0.2 & 0.1 \\
$\hat{D} \xi_{\mathrm{p} \varphi}(\mathrm{cm})$ & 5 & 6 & 8 & 8 & 9 & 10 & 12 \\
\hline
\end{tabular}


and maximum sea levels have normal distributions and are independent, hence their total effect is given by:

$$
\hat{D} \xi_{T_{p \varphi \alpha}}=\sqrt{\hat{D}_{T_{\alpha}}^{2}+\hat{D}^{2} \xi_{\mathrm{p} \varphi}}
$$

where $\hat{D} \xi_{\mathrm{p} \varphi}=$ upper limit of the confidence interval for quantile $\hat{\xi}_{p \varphi}$ (see Table 4 ), and $\hat{D}_{T_{\alpha}}=$ upper limit of the confidence interval for the sea level forecast error (see Eq. 10).

Eqs (17) \& (18) enable the exceeding probability quantile of the maximum sea level for time $T$ to be computed. The simple superposition of the trend forecast and detrended maximum sea level is presented by:

$$
\hat{\xi}_{T_{p \varphi}}=\hat{\xi}_{p \varphi}+\mathbf{c}^{\prime} \hat{\mathbf{a}}_{T \psi}
$$

where $\hat{\xi} \bullet_{p \varphi}=$ the maximum sea level at time $T$ as the superposition of the trend forecast (reference level $\hat{\boldsymbol{a}}_{0}$ ) and $\hat{\xi}_{p \varphi}$.

The forecast computation was performed for the 0.001 quantile for the period 1990 to 2100; Eq. (11) was taken into account. The results are set out in Fig. 4.

\section{CONCLUSIONS}

Extrapolated over a long period of time, the mean sea level forecast has a broad interval of uncertainty. Such a forecast for engineering purposes requires a method accounting for the so far determined linear sea level rise. The use of a trend-extrapolated forecast requires that confidence intervals be extensively applied, which enables the method to be adapted to the various sea level rise scenarios compiled by IPCC. The choice of confidence levels under these conditions is subjective, as is the choice of return period and confidence levels in the sea level storm surge probabilistic computations.

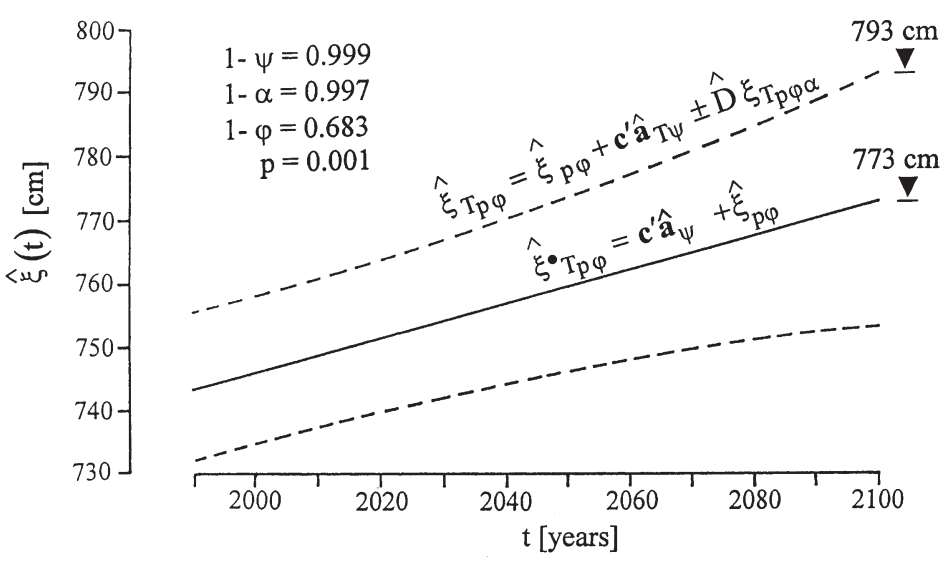

Fig. 4. Forecast of maximum sea levels at Kołobrzeg up to 2100 according to Eqs (18) \& (19)
The application of confidence intervals to the prediction of mean and maximum sea levels facilitates the addition of 2 forecasts, the result of which is less than simple superposition (see Eq. 18).

The computation method enables a sea level forecast for tide-gauges with various trends to be made at the same level of confidence. In this case, engineering designs would need to take into account only the local trend and the same confidence levels for the whole coast. Example computations were performed for the Kołobrzeg tide-gauge readings.

\section{LITERATURE CITED}

Bardsley WE, Mitchell WM, Lennon GW (1990) Estimating future sea level extremes under conditions of sea level rise. Coastal Eng 14:295-303

Douglas BC (1991) Global sea level rise. J Geophys Res 96(C4):69814-6992

Douglas BC (1992) Global sea level acceleration. J Geophys Res 97(C8):12699-12706

Draper NR, Smith H (1981) Applied regression analysis. Wiley, New York

Dziadziuszko Z (1994) Annual mean and maximum sea levels in Polish harbours. Institute of Meteorology and Water Management, Maritime Branch, Gdynia (in Polish)

Fisher RA, Tippet LHC (1928) Limiting forms of the frequency distribution of the largest or smallest member of a sample. Proc Camb Phil Soc 24:180-190

Gornitz V, Solov A (1991) Observations of long-term tide gauge records for indicators of accelerated sea level rise. In: Schlesinger ME (ed) Greenhouse gas-induced climatic change: a critical appraisal of simulations and observations. Elsevier, Amsterdam, p 347-367

Gumbel EJ (1958) Statistics of extremes. Columbia University Press, New York

Jenkinson AF (1955) The frequency distribution of the annual maximum (or minimum) values of meteorological elements. Q J R Met Soc 81:158-171

Kimball BF (1949) An approximation to the sampling variance of an estimated maximum value of given frequency base on fit of double exponential distribution of maximum values. Ann Math Stat 20:110-113

Pugh DT (1987) Tides, surges and mean sea-level. Wiley, New York

Snedecor GW, Cochran WG (1967) Statistical methods. University Press, Iowa State University, Ames, IA

Tushingham AM, Peltier WR (1991) ICE-3G: a new global model of late Pleistocene deglaciation based upon geophysical predictions of post glacial relative sea level change. J Geophys Res 96B:4497-4523

Warrick RA, Le Provost C, Meier MF, Oerlemans J, Woodworth PL (1996) Changes in sea level. In: Climate Change 1995; Contribution of Working Group I to the Second Assessment Report of IPCC. Cambridge University Press, Cambridge, p 358-405

Woodworth PL (1990) A search for acceleration in records of European mean sea level. Int J Climatol 10: 129-143

Wróblewski A (1994) Analysis and long-term forecast of sea levels along the Polish Baltic Sea coast Part II. Annual mean sea levels - forecast to the year 2100. Oceanologia 36(2):107-120

Zelias A (1984) Theory of forecast. St Econ Publ, Warsaw 